\title{
Oassin \\ GPR120 dysfunction can cause obesity in mice and humans
}

A mutation that affects signaling by the long-chain free fatty acid receptor GPR120 leads to obesity, according to an international research group. Mice lacking GPR120 developed obesity and humans who had a nonsignaling variant of the receptor were prone to obesity.

The GPR120 knockout mouse model was developed by a team in Japan who studied weight changes in these mice when they were fed a normal or high-fat diet. "The reason for us to focus on weight was that GPR120 is abundant in the gut and adipose tissue," explains Gozoh Tsujimoto, corresponding author.

Knockout mice fed a high-fat diet developed insulin resistance, had heavier white adipose tissue and livers, as well as increased plasma levels of cholesterol and raised serum levels of alanine aminotransferase (indicating impaired cholesterol metabolism and liver function), compared with knockout mice fed a normal diet.

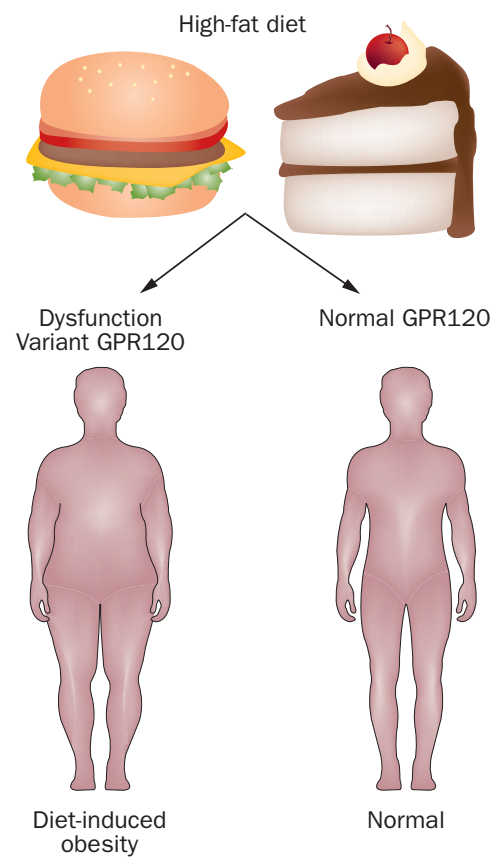

People with dysfunctional GPR120 are prone to diet-induced obesity. Image produced in consultation with G. Tsujimoto.
To investigate GPR120 function in humans, the Japanese team collaborated with international researchers to carry out genetic studies of 6,942 obese and 7,654 nonobese people. They found a GPR120 variant (p.R270H) that lacked the ability to transduce signals of long-chain free fatty acids. This variant was associated with obesity.

"Genotyping of GPR120 could lead to personalized medicine," says Tsujimoto. As such, people with the nonsignaling p. $\mathrm{R} 270 \mathrm{H}$ variant could be identified as being at risk of obesity and encouraged to adopt a low-fat diet. In addition, the researchers suggest that novel antiobesity drugs could be developed to target GPR120.

Andy McLarnon

Original article Ichimura, A. et al. Dysfunction of lipid sensor GPR120 leads to obesity in both mice and human. Nature doi:10.1038/nature10798 